\title{
Predictive maintenance of photovoltaic plants using multirotor drones with automatic recharging system of Li-Po batteries
}

\author{
Vasile Pleşca ${ }^{1 *}$, and Cristian Purece ${ }^{2}$ \\ ${ }^{1}$ Politehnica University of Bucharest, Electrical Engineering PhD School, Bucharest, Romania \\ ${ }^{2}$ INCDE ICEMENERG, Bucharest, Romania
}

\begin{abstract}
Creating the correct maintenance of the strings that form the electric generators, from a photovoltaic park, it requires scanning of the equipment with thermal vision cameras, in the visible and infrared field, at an optimum angle determined precisely for each location depending on the geographic position and the architecture of the power station. In general, this angle is difficult to maintain from the ground so it is necessary to mount the cameras on a support, or most efficiently on a multi-rotor drone that is very stable and easy to handle. Maintenance using multi-rotor drones and heat sensitive cameras is no longer a novelty and has multiple advantages: low costs, minimization of work accidents, and the data acquired can be analyzed in real time or can be stored for later analysis. But this process also has disadvantages: limited flight autonomy of drones, generated by the hard development of battery technology and a small number of specialized personnel in this field. To minimize these disadvantages, we have developed an automatic recharging system of batteries, without disconnecting them, which allows the automation of the scanning process and offers the possibility of taking control of the drones and from a distance.
\end{abstract}

\section{Introduction}

Romania has an estimated solar energy potential of 1433 thousand TEP, the equivalent to $60 \times 106 \mathrm{GJ} /$ year and 103.2 thousand TEP electricity, which is about $1200 \mathrm{GWh} /$ year. Due to its geographical position and the diversified structure of the relief forms, Romania is well suited to all renewable energy sources, and from the point of view of solar energy, our country has a potential with over $60 \%$ sunny days, that is 210 days out of a total year, almost $20 \%$ better than Germany, which has long been a leader in the field. $[4,6,8,10]$

According to Transelectica, 962 photovoltaic parks were built in Romania between 2013 and 2017, representing approximately 4,871.66 MW installed power. Statistically, of these, 212 have a power installed below $1 \mathrm{mw}$, and 112 have a power installed below two mw. On development regions, the South-Muntenia region has the most photovoltaic parks

* Corresponding author: adi plesca@yahoo.com 
and the highest installed power, being followed by the Central and North-West region. On the counties, the first places are owned by Brasov, Prahova and Giurgiu counties, but when it comes to the installed power, Giurgiu owns the first place, with an installed power of 79.2 MW, followed by Brasov, with an installed power of $61 \mathrm{MW}$ and Calarasi $60 \mathrm{MW}$. From the point of view of the surface occupied by the photovoltaic park, the average is between $20 \div 50$ hectares, and the largest parks are found in Sebiş - Arad, with an area of 202 ha, Recaş - Timiş with an area of 200 ha and Livada - Satu Mare with an area of 135 ha. [6, 8, $10,11]$.

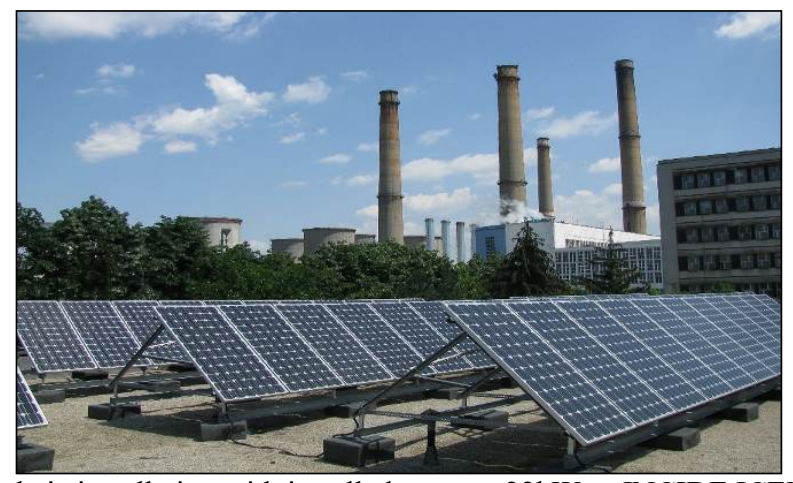

Fig. 1. Photovoltaic installation with installed power - 22kWp - INCIDE ICEMENERG [15]

As all these photovoltaic parks are approaching half their lifetime, depending on the quality of the installed photovoltaic panels, concrete measures are required to manage the possible defects that are assimilated with this type of plants. Thus, performing predictive maintenance can limit yield losses and prevent escalation of defects from one equipment to another or from one area to another. The previous picture shows a small installation, located on the terrace of the laboratory body, of INCDE ICEMENERG, with PIF in 2007, in which tests were performed on its equipment.

\section{Complex system for the maintenance of photovoltaic power plants}

Through the method of interconnecting the cells at the panels, the panels in strings and the strings in the inverter systems, during the exploitation of this equipment, a series of defects may appear, some easy to see with the naked eye but others difficult to identify, practically only when the defect has already caused major losses by propagating to other equipment or generating fires with a negative impact on the entire plant or its vicinity.

Because these installations are usually distributed on rather large surfaces or at great heights, in the case of those installed on the roof, without any special equipment, normal or preventive maintenance it is very difficult to achieve this. Many of the defects can be determined by other comparative methods but these are enough that if they are not detected and remedied in a short time from their emergence, they can generate other defects with a much greater impact.

\subsection{General notions used in the maintenance process}

Generally, in a photovoltaic installation, the defects have as a secondary effect in the first phase, a local overheating. Thus, a scan with thermal imaging cameras, at the optimum angle and distance, generally detects $99 \%$ of the defects. Due to the size of the photovoltaic 
power plants and the large number of elements to scan, the manual inspection is not very efficient so it is necessary to scan from air as well, at the same time capturing images in the IR and visible spectrum.

For aerial scanning you can use several methods or better said more aircraft: with human crew, various types of drones but also balloons properly equipped. Analyzing these variants it becomes obvious that the balloon is excluded from the beginning because the time required for a complete scan is very large but also because it would be very difficult to keep the same trajectory and optimal distance from the surface of the panels, at the next scan, a useful function for comparing the captured images and issuing a diagnosis as close to reality as possible. The variant of flying equipment with human crew is an option, but it has some disadvantages that are difficult to overlook. These are expensive and the captured images are made from a rather large distance $(30 \div 50 \mathrm{~m})$, the distance that introduces errors in the final analysis because not all the defects are highlighted. Thus, the only viable solution are drones that have the ability to scan automatically or remotely, on predetermined routes or at predefined angles, and the cost of such a service is much lower than the option of using a solution with human personnel on board. Although this solution has many advantages overall, it also has a number of disadvantages: the speed of flight is slower than the version with human personnel on board but it compensates by obtaining images with much better resolutions. However, the biggest disadvantage of the drones is their low autonomy, so the total scan time of the entire park will be quite long taking a few minutes to recharge / change such a battery.

Because such scanning equipment does not cost very much compared to a humanpowered aircraft, or relative to the rest of the equipment in a facility, each park can afford itself to purchase such a system. In addition, the operation of such equipment can be done automatically by creating points in the area of the park and where the drone is desired to be controlled by the operating personnel with a minimum of training. In this case, the flight authorizations are obtained very easily, because the flight height does not exceed $30 \mathrm{~m}$ and in general the locations of these stations are located in the outer-urban environment.

A complex system for scanning photovoltaic parks is composed of:

- multirotor drone support;

- high resolution visible camera;

- thermal imaging camera;

- system of storage and processing acquired data, located inside the park or at a distance;

- system of automatic recharging of the batteries located in the center of the park, preferably near an inverter system that provides the necessary energy and the efferent communications with the rest of the systems;

- digital weather station;

\subsection{Thermal-vision cameras used to scan photovoltaic panels}

In general, the companies specialized in the production of thermal-vision cameras also have dual cameras which include an IR sensor but also a high-resolution sensor in the visible spectrum. $[12,13]$ Thus, the scanning process, but especially the processing of the acquired data, is a much faster and more accurate one, as the camera can eliminate a series of scanning errors automatically. If no such equipment is available, a high-resolution camera can be used, in parallel with a thermal-vision one, and by superimposing the two visible and thermal-vision spectra and using specific algorithms for anomalous detection, through the overlapping of acquired images and comparisons with control images from the database, we can identify a number of differences that may have as a cause the following defects:

- defective cells at the level of a photovoltaic panel; 
- photovoltaic panels in short circuit;

- weak connections at the level of strings or blocks of connections;

- defects in the connection cables due to mechanical, accidental actions;

- pollution level by identifying deposits on the surface of photovoltaic panels, which can be: dust layer or chemical, industrial deposits from industry in the area;

- cracks of the protective surface or cells of a photovoltaic panel;

- possible problems due to total or partial shading due to the vegetation in the location or from the layers of snow, frost, ice deposited on the active surface of the panels.
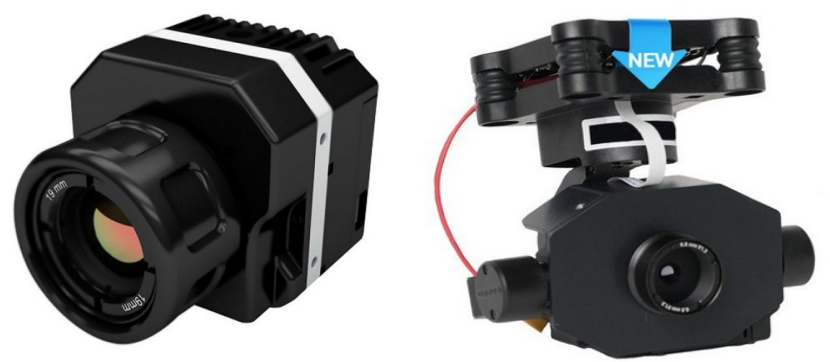

Fig. 2. Thermal imaging camera specific for scanning photovoltaic panels, in simple form or mounted on a gimbal. [12]

For an optimal inspection of a photovoltaic panel using the thermal-vision scanning system, there must be a solar radiation of $500 \mathrm{~W} / \mathrm{m}^{2}$, preferably as high as possible, without variations greater than $100 \mathrm{~W} / \mathrm{m}^{2}$ during the measurements, so the cloudy days but especially the windy days should be avoided because it randomly changes the thermal gradient on the active surface of the panel. Also, in order to easily highlight the temperature differences at the panel level, a thermal sensitivity of less than $0.08 \mathrm{~K}$ is required. During the summer, these conditions are quite easy to reach, because the days are longer, but in the case of frosty winter days, an optimal scan can be done only a few tens of minutes a day, in which case the process takes quite a long time. If these conditions can not be met, the scanning process is slow, which can not be done automatically because too many errors occur and uncertainties. Thus, the specialist who operates the scanning system must be present at the location and eliminate any errors that may occur through repeated scans at that point.

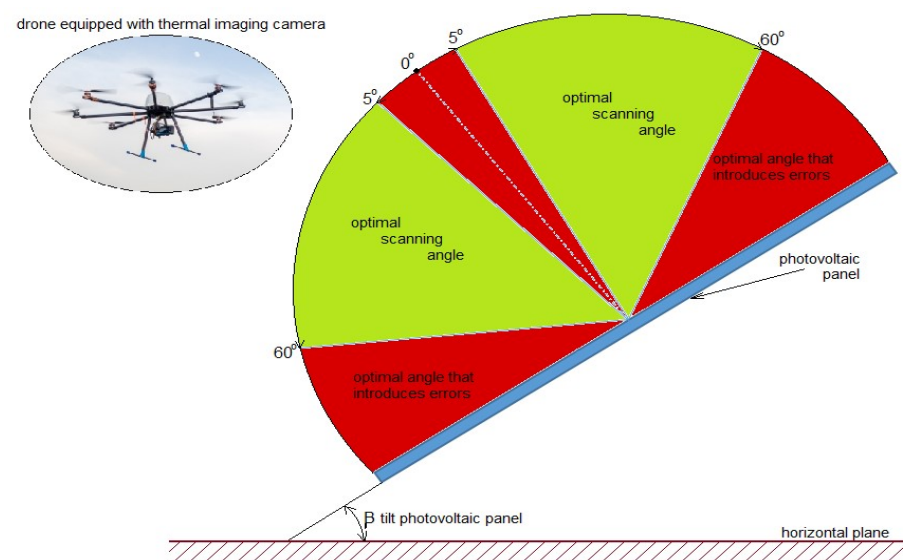

Fig. 3. Determining the optimum angle for scanning the photovoltaic panels with thermal imaging camera 
In order to avoid occasional reflections and shadows that can return images with false defects, during the scanning process, it is recommended that the angle of passage not be perpendicular to the surface of the panel and be indicated within in the ranges: $5 \div 60$ degrees or $-5 \div-60$ degrees, compared to the perpendicular formed with the surface of the panel, avoiding the interval \pm 5 degrees (fig. 3 ).

The minimum resolution of the thermal imaging camera must be better than $640 \times 512$ physical pixels, and the recording frequency of the images $8.3 \mathrm{~Hz}$ PAL or $7.5 \mathrm{~Hz}$ NTSC. If a better scan frequency is desired on the market there are also a variant of $25 \mathrm{~Hz}$ PAL and 30 $\mathrm{Hz}$ NTSC but for this you need a license. Obviously, since we are talking about a camera that is mounted on a drone, every gram counts so the camera along with the associated lens is advised not to exceed 150 grams, and the dimensions to be as compact as possible. Very important is its instantaneous consumption which is preferable to be below $2 \mathrm{~W}$ and at a voltage around $5 \mathrm{Vcc}$. Also, both cameras must be mounted solidly on a gimbal that must ensure mechanical stability by eliminating vibrations and that can be controlled fine from a distance. Also, from a distance, access to the entire menu of the cameras must be ensured so that optimum optical and / or digital zoom can be achieved, including setting the main parameters during the scan: color palettes, camera mode, camera optimization, polarity control, etc. Thus, the camera must be $100 \%$ compatible with the autopilot system of the drone, but also with the telemetry system installed on it. $[4,9,12,13]$
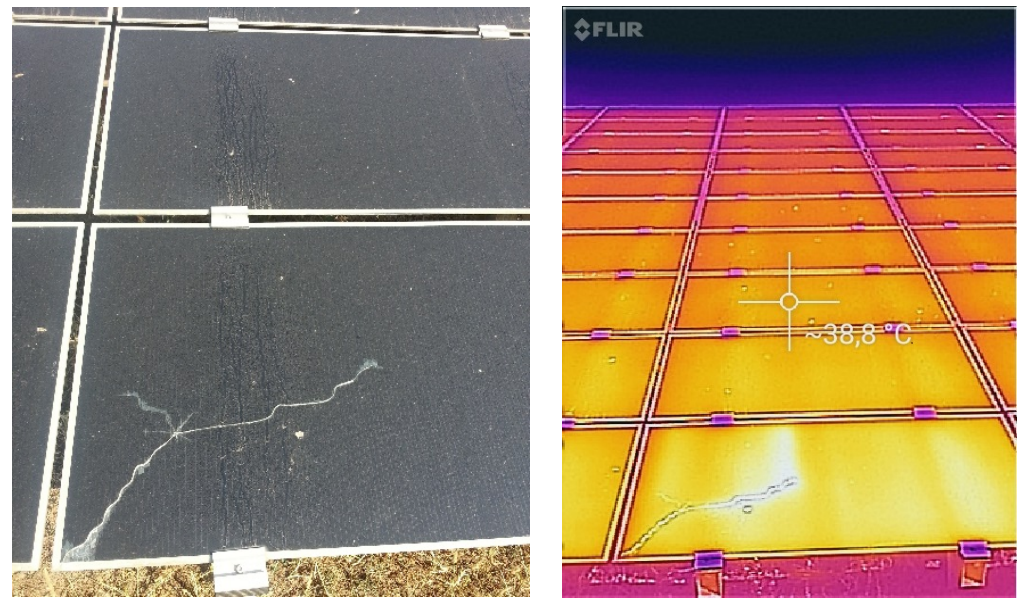

Fig. 4. Crack in the photovoltaic panel seen in the visible and infrared spectrum - Mavrodin photovoltaic plant [15]
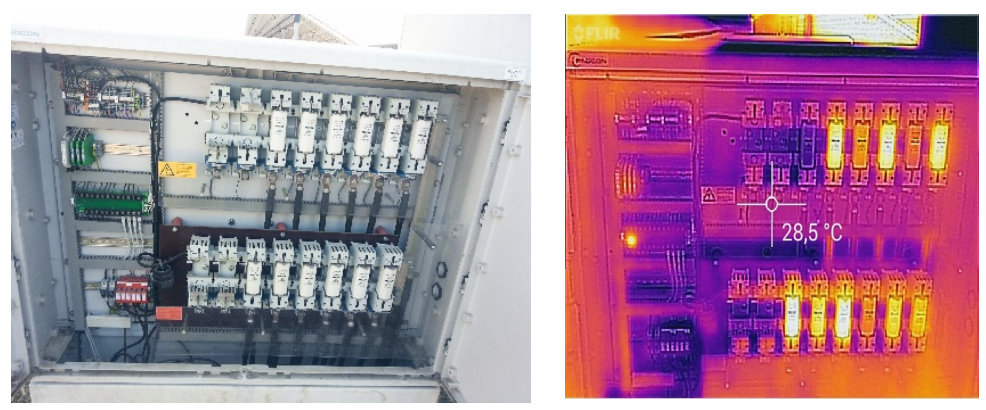

Fig. 5. Exposure of the connections panel in the visible and infrared spectrum - Mavrodin photovoltaic plant [15] 


\subsection{Drone selection as scanning system support}

Depending on the weight of the sensors and the size of the photovoltaic park, the support drone can be one with a quad-copter, hex-copter or octo-copter type, with four or eight arms. For this to be able to operate autonomously and to follow the geo-referential map of the photovoltaic park, it must be equipped with a high precision GPS sensor, of the order of centimeters on the horizontal but also on the vertical. Data registration will be done at the drone level but will also be transmitted remotely to a database and a processing server. In addition, the telemetry system installed on the drone will retrieve data from the autopilot and video cameras and transmit it to the ground: GPS position, flight speed, altitude, instantaneous battery capacity and camera status; an example of such a drone can be seen in the following figure. The ground processing unit estimates based on the data received from the drone, collaborated with the data from the weather system located in the park, the drone deviation from the predetermined route for scanning and the estimation of the remaining flight time. When the estimated flight time or battery capacity falls below pre-set thresholds, the system sends the drone a return command to the docking station where the battery recharging process will begin. When the recharging process is complete, the system tests all parameters, including weather data and if all is well, it sends the drone an order to restart the scanning process from where it left off, taking into account the new position of the Sun.

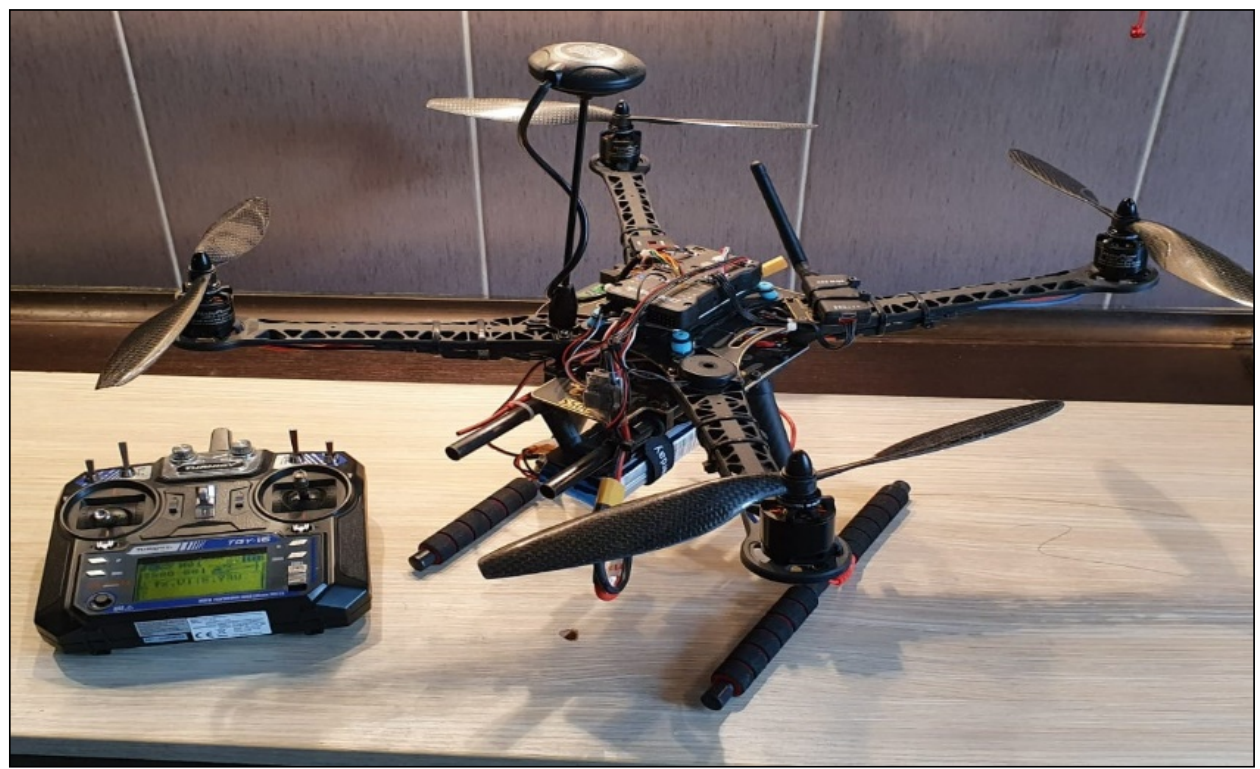

Fig. 6. Quad-copter type drone used during testing [15]

During the scan, the system analyzes the acquired data and if the anomalies appear on the image, they mark the place on the geo-referential map. An operator will determine the type of defect, the priority of remedy and the analysis of the cause that caused the defect. Defects are identified using specialized algorithms that take into account the references previously registered in the databases: dimension, location and spectral references. $[1,4,9$, $12,13]$. 
Table 3: Classifying the defects according to the spectral images at a new photovoltaic panel

\begin{tabular}{|l|l|l|}
\hline Image recorded & Possible cause & Possible damage \\
\hline \multirow{3}{*}{ Hot Spot } & Dust deposit & \multirow{2}{*}{$\begin{array}{l}\text { Temporary coverage of the } \\
\text { active area }\end{array}$} \\
\cline { 2 - 2 } & $\begin{array}{l}\text { Layer deposited due to local } \\
\text { pollution }\end{array}$ & \multicolumn{1}{|c|}{ Production defects } \\
\cline { 2 - 3 } & Deposit of bird droppings \\
\cline { 2 - 2 } & Excessive humidity & \\
\hline Hot spot & Cracks of the cells & $\begin{array}{l}\text { Production defects, cracks of } \\
\text { cells, short circuit and bypass } \\
\text { diode defect }\end{array}$ \\
\hline Hot spot / cold spot & $\begin{array}{l}\text { Impurities or bubbles of gas on } \\
\text { the sandwich panels }\end{array}$ \\
\hline panel & $\begin{array}{l}\text { Cracks on the surface of the } \\
\text { panel at the level of a cell/ or than the rest of the } \\
\text { several cells }\end{array}$ \\
\hline $\begin{array}{l}\text { Photovoltaic panels or strings } \\
\text { hotter the rest }\end{array}$ & $\begin{array}{l}\text { Electrical contact problems or } \\
\text { the panels are not connected }\end{array}$ & $\begin{array}{l}\text { Mounting defects or electrical } \\
\text { defects }\end{array}$ \\
\hline
\end{tabular}

\subsection{Automatic battery recharging system}

The automatic reloading system is a prototype made especially for multi-engine drones that perform missions independently. The system is a fixed one, based on a series of sensors and two microcontrollers, one located on a charging base and one at the drone level.

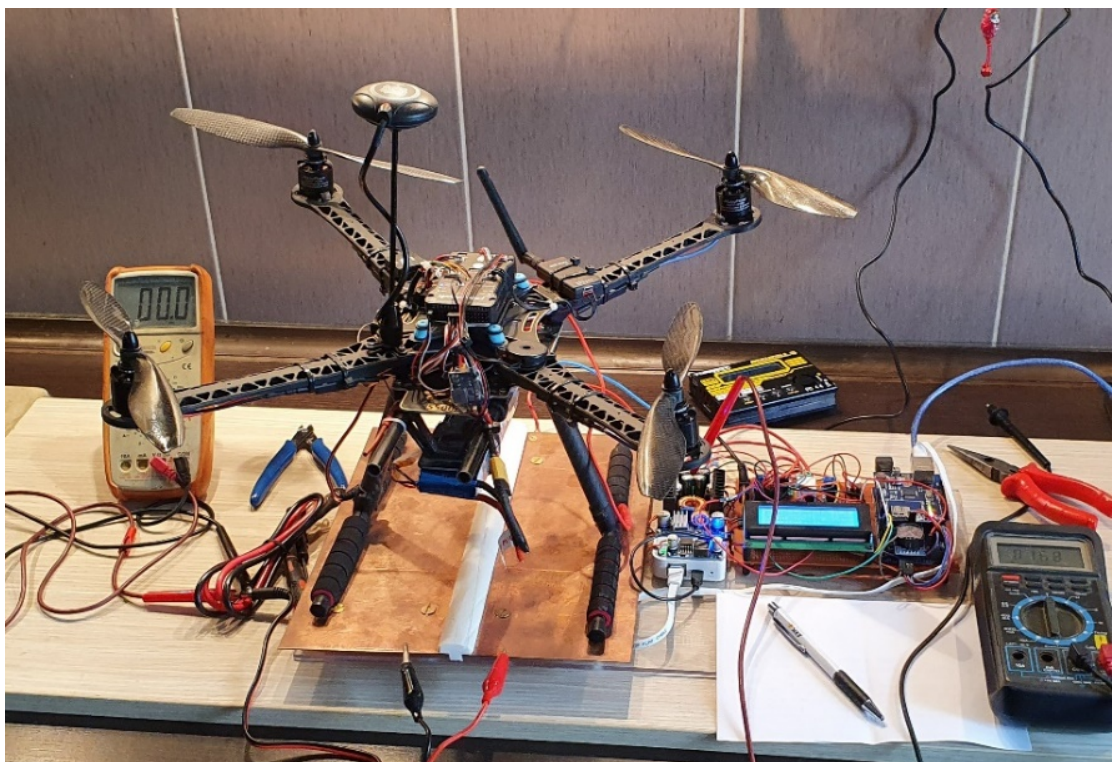

Fig.7. Experimental automatic charging system of the monitoring drone batteries [15]

When the drone arrives for recharging, the two microcontrollers connect to perform the steps required to perform the task:

- fixed landing with precise positioning in the horizontal plane. At the drone level and on a fixed base, special sensors are mounted for the precise positioning of the drone on the loading area;

- drone status check after landing confirmation - zero current passed on the main path (engines stopped). This eliminates any confirmations that may appear wrong and which may lead to the system crashing; 
- battery polarity testing / synchronization. In the event of a landing in strong wind conditions, the position of the drone on the loading base may be different from that established, and the probes may be in the wrong position. Risk of battery explosion and drone system damage. If there is an error, it will wait until there are flight conditions and restart the drone on the basis of loading. If everything goes normally the system starts the charging process;

- start of the process of charging the batteries with the tracking of charging curve specified in the documentation;

- testing / balancing battery cell voltage throughout the charging process;

- when the system confirms the full charge of the battery, the weather conditions and the hour set for the scan are tested. If all the required conditions are met the drone takes off and resumes the scan process from the last position, taking into account the position of the Sun.

The contact between the polarized loading pads and the drone is made by a probe system, mounted on the drone landing gear, which, using the weight of the entire dronemounted equipment, helps to make a firm contact.

\subsection{Weather station}

The digital weather station must be minimally equipped with anemometer, wind vane, pyranometer, thermometer and hygrometer and must return results in digital format with an accepted accuracy: wind speed and direction, solar radiation, temperature and humidity of the environment. If the photovoltaic park has a large surface area or if there are large differences in level, the presence of several sensors to eliminate errors is required.

\section{Conclusions}

The increasing number of new photovoltaic installations, installed in Romania, but especially the large number of applications at maturity, with an operating age of approximately 7 years, makes the predictive maintenance service still in high demand from now on. The use of multi-engine drones equipped with heat-sensitive cameras for maintenance of such installations is no longer a novelty, but it has been proven in time that it is a cheap and reliable solution that can detect early defects that cannot be identified with other monitoring equipment. Thus, an acceptable yield is maintained at the level of the entire installation, and the beneficiary has minimal losses in the operation of the respective installation, maintaining the operating costs within acceptable limits.

By automating the entire scanning process there are several advantages:

- eliminates any human errors that may occur due to the routine or the conditions in the site; - the optimal positioning of the video equipment relative to the elements to be scanned depends on the position of the Sun and the angle of the panels with horizontal;

- automatic limitation of the scans depending on the weather conditions: wind speed, precipitation, solar radiation level, the state of the plant and the level of charge of the power flowed into the system.

- reducing maintenance costs by automatically performing predictive maintenance;

- increasing or maintaining the efficiency of the photovoltaic power plant by identifying in a timely manner the defects that have occurred without reaching major defects with interruptions in the partial or total functioning of the power plant;

The work has been funded by the Operational Programme Human Capital of the Ministry of Europe Funds through the Financial Agreement 51675/09.07.2019, SMIS code 125125. 


\section{References}

1. Yevgen Barsukov, Jinrong Qian - Battery Power Management for Portable Devices, ISBN 13: 978-1-60807-491-4, C 2013 ARTECH HOUSE 685 Canton Street Norwood, MA 02062 (2013)

2. Kimon P. Valavanis, John Evans, Daniel Felix, George J. Vachtsevanos - "Handbook of Unmanned Aerial Vehicles", ISBN: 978-90-481-9706-4, 978-90-481-9707-1, Springer Science + Business Media Dordrecht (2015)

3. B. Clough, Metrics, schematics! How do you track a UAV's autonomy? In Proceedings of the AIAA 1st Technical Conference and Workshop on Unmanned Aerospace Vehicles, Portsmouth, (2002)

4. Ghe. Olteanu, V. Plesca and others - Energy in the post-oil era. ISBN 978-973-174123-9, editura ICEMENERG (2010)

5. 1625-2008 - IEEE Standard for Rechargeable Batteries for Multi-Cell Mobile Computing

Devices - IEEE 1625-2008 -

https://ieeexplore.ieee.org/stamp/stamp.jsp?tp=\&arnumber=4657368 (2020)

6. https://appsso.eurostat.ec.europa.eu/nui/show.do?dataset=nrg_ind_ren\&lang=en (2020)

7. https://www.autogreen.ro/energie/nivelul-de-insolatie-si-dimensionare-panourilor/ (2019)

8. https://ec.europa.eu/eurostat/statistics-

explained/index.php?title=Renewable_energy_statistics/ro (2019)

9. http://solarcenter.ro/blog/panourile-solare-mod-de-functionare-si-beneficii/ (2019)

10. http://free-energy-monitor.com/index.php/energy/fotovoltaice (2019)

11. http://www.transelectrica.ro (2020)

12. https://www.flir.com/products/vue-pro-r/ (2020)

13. https://www.dronebase.it/prodotto/dual-sensor-ir-eo-gimbal-gemini-ir-2/ (2020)

14. http://www.dummies.com/consumer-electronics/drones/battery-considerations-foryour-drone/ (2020)

15. Personal photos 